\title{
Exponential Jacobi spectral method for hyperbolic partial differential equations
}

\author{
Y. H. Youssri ${ }^{1}$ - R. M. Hafez ${ }^{2}$ \\ Received: 5 May 2018 / Accepted: 16 September 2019 / Published online: 26 September 2019 \\ (c) The Author(s) 2019
}

\begin{abstract}
Herein, we have proposed a scheme for numerically solving hyperbolic partial differential equations (HPDEs) with given initial conditions. The operational matrix of differentiation for exponential Jacobi functions was derived, and then a collocation method was used to transform the given HPDE into a linear system of equations. The preferences of using the exponential Jacobi spectral collocation method over other techniques were discussed. The convergence and error analyses were discussed in detail. The validity and accuracy of the proposed method are investigated and checked through numerical experiments.
\end{abstract}

Keywords First-order partial differential equations · Exponential Jacobi functions · Operational matrix of differentiation · Heisenberg matrix $\cdot$ Convergence analysis

Mathematics Subject Classification 35L02 $\cdot 65 \mathrm{M} 70$

\section{Introduction}

Hyperbolic partial differential equations (HPDEs) constitute an important subclass of partial differential equations. The HPDEs are used in many disciplines of science and engineering, such as studying the transmission and propagation of electrical signals [1], wave propagation [2], hypoelastic solids [3], astrophysics [4], process engineering [5], acoustic transmission [6] and random walk theory [7]. The HPDEs are used in shaping the vibrational motion of structures (e.g., beams, machines and buildings) and represent basis for fundamental equations of atomic physics [8, 9]. Recently, the study of exact and numerical solutions of either hyperbolic or parabolic PDEs has received increasing attention [10-15].

Spectral techniques have been successfully applied for approximating the solution of differential problems defined in unbounded domains. For problems with sufficient smooth analytic solutions, they exhibit exponential rates of convergence, high accuracy and low computational cost.

Y. H. Youssri

youssri@sci.cu.edu.eg

1 Department of Mathematics, Faculty of Science, Cairo University, Giza 12613, Egypt

2 Department of Mathematics, Faculty of Education, Matrouh University, Matrouh, Egypt
Doha et al. [16] used a Jacobi rational spectral technique for solving Lane-Emden initial value problems, in astrophysics, on a semi-infinite interval. Hafez et al. [17] applied a new collocation scheme for solving hyperbolic equations of second order in a semi-infinite domain. Doha et al. [18] proposed a new spectral Jacobi rational-Gauss collocation method for solving the multi-pantograph delay differential equations on the half line. Bhrawy et al. [19] solved some higher order ordinary differential equations using a new exponential Jacobi pseudospectral method.

In this study, we used exponential Jacobi functions for numerically solving the HPDEs. The operational matrices of derivatives and products of exponential Jacobi functions were derived. These matrices were jointly implemented with the collocation approach to evaluate the solutions of the HPDEs. Collocation method [20-24] is an effective technique for numerically approximating different kinds of equations.

The workflow of this paper encompass: In the next section, we present some notations and other mathematical facts. "Operational matrix of differentiation for exponential Jacobi" section is devoted to the operational matrix of differentiation for exponential Jacobi functions. In "Implementation of the method" section, the operational matrix of differentiation for exponential Jacobi was used in a combination with the exponential Jacobi collocation method to solve the HPDEs. The 
error analysis was executed in "Error analysis" section. Two numerical examples are given in "Numerical results" section. Finally, some concluding remarks are mentioned in "Conclusion" section.

\section{Mathematical preliminaries}

Here, we list some useful mathematical relations and identities needed in the construction of the exponential Jacobi operational matrix.

\section{Exponential Jacobi functions}

Consider the standard classical Jacobi polynomials $J_{k}^{(\rho, \sigma)}(z)$ on the interval $[-1,1]$ with the weight function $\omega^{(\rho, \sigma)}(z)=(1-z)^{\rho}(1+z)^{\sigma}, \rho, \sigma>-1$,

$J_{0}^{(\rho, \sigma)}(z)=1, \quad J_{1}^{(\rho, \sigma)}(z)=\frac{1}{2}(\rho-\sigma+z(\rho+\sigma+2))$,

the set $\left\{J_{k}^{(\rho, \sigma)}(z): k=0,1, \ldots\right\}$ forms a complete orthogonal system in the weighted Hilbert space $L_{\omega^{\rho, \sigma}(x)}^{2}[-1,1]$ equipped with the inner product

$(f, g)_{\omega^{(\rho, \sigma)}(x)}:=\int_{-1}^{1} f(x) g(x) \omega^{(\rho, \sigma)}(x) d x$,

and the norm

$\|f\|_{\omega^{(\rho, \sigma)}(x)}=(f, f)_{\omega^{(\rho, \sigma)}(x)}^{\frac{1}{2}}$.

Let us define the exponential Jacobi functions by replacing $z$ by $1-2 e^{-\frac{x}{L}}$. Denoting the exponential Jacobi functions $J_{i}^{(\rho, \sigma)}\left(1-2 e^{-\frac{x}{L}}\right)$ by $\Upsilon_{i}^{(\rho, \sigma)}(x), x \in[0, \infty)$. Therefore, $\Upsilon_{i}^{(\rho, \sigma)}(x)$ may be generated by the following recurrence relation:

$$
\begin{aligned}
& \Upsilon_{k+1}^{(\rho, \sigma)}(x)=\frac{(2 k+\rho+\sigma+1)(2 k+\rho+\sigma+2)}{(k+1)(k+\rho+\sigma+1)} \\
& \quad\left[\left(\frac{\left((\rho+1)(\rho+\sigma)+2 k^{2}+2 k(\rho+\sigma+1)\right)}{(2 k+\rho+\sigma)(2 k+\rho+\sigma+2)}-e^{-\frac{x}{L}}\right) \Upsilon_{k}^{(\rho, \sigma)}(x)\right. \\
& \left.\quad-\frac{(k+\rho)(k+\sigma)}{(2 k+\rho+\sigma)(2 k+\rho+\sigma+1)} \Upsilon_{k-1}^{(\rho, \sigma)}(x)\right], \quad k \geq 1,
\end{aligned}
$$

where

$\Upsilon_{0}^{(\rho, \sigma)}(x)=1, \quad \Upsilon_{1}^{(\rho, \sigma)}(x)=(\rho+1)-(\rho+\sigma+2) e^{-\frac{x}{L}}$,

and

$(k+\rho+\sigma) \Upsilon_{i}^{(\rho, \sigma)}(x)=(k+\sigma) \Upsilon_{i}^{(\rho, \sigma-1)}(x)+(k+\rho) \Upsilon_{i}^{(\rho-1, \sigma)}(x)$.

The exponential Jacobi functions $\Upsilon_{i}^{(\rho, \sigma)}(x)$ of degree $i$ can be written as
$\Upsilon_{i}^{(\rho, \sigma)}(x)=\sum_{k=0}^{i}(-1)^{k} \frac{\Gamma(i+\rho+1) \Gamma(i+k+\rho+\sigma+1)}{\Gamma(\rho+k+1) \Gamma(i+\rho+\sigma+1)(i-k) ! k !} \exp (-k x / L)$,

where

$\Upsilon_{i}^{(\rho, \sigma)}(0)=\frac{(-1)^{i} \Gamma(\sigma+i+1)}{i ! \Gamma(\sigma+1)}$.

The set $\left\{\Upsilon_{i}^{(\rho, \sigma)}(x): i=0,1, \ldots\right\}$, satisfy the following orthogonality relation:

$\int_{0}^{\infty} \Upsilon_{i}^{(\rho, \sigma)}(x) \Upsilon_{j}^{(\rho, \sigma)}(x) w^{(\rho, \sigma)} d x=h_{i}^{(\rho, \sigma)} \delta_{i j}$

where

$w^{(\rho, \sigma)}=e^{-\frac{\rho+1}{L} x}\left(1-e^{-\frac{x}{L}}\right)^{\sigma}$,

$h_{i}^{(\rho, \sigma)}=\frac{L \Gamma(i+\rho+1) \Gamma(i+\sigma+1)}{i !(2 i+\rho+\sigma+1) \Gamma(i+\rho+\sigma+1)}$,

and $\delta_{i j}$ is the well-known kronecker delta.

\section{Function approximation}

Now, approximation of $u(x)$ by $N+1$ terms of exponential Jacobi functions yields

$u(x) \simeq \sum_{j=0}^{N} c_{j} \Upsilon_{j}^{(\rho, \sigma)}(x)=\mathbf{C}^{T} \phi(x)$,

where $\mathbf{C}$ and $\phi(x)$ are the unknown coefficients vector and the exponential Jacobi function vector, respectively, and are given by:

$\mathbf{C}=\left[c_{0}, c_{1}, \ldots, c_{N}\right]^{T}$,

$c_{i}=\frac{1}{h_{i}^{(\rho, \sigma)}} \int_{0}^{\infty} u(x) \Upsilon_{i}^{(\rho, \sigma)}(x) w^{(\rho, \sigma)} d x$,

and

$\phi(x)=\left[\Upsilon_{0}^{(\rho, \sigma)}(x), \Upsilon_{1}^{(\rho, \sigma)}(x), \ldots, \Upsilon_{N}^{(\rho, \sigma)}(x)\right]^{T}$

\section{Operational matrix of differentiation for exponential Jacobi}

Here, we report the derivation of the operational matrix of derivatives of the exponential Jacobi functions, which is of important use to our numerical scheme. 
Theorem 1 Let $\phi(x)$ be the exponential Jacobi vector defined in (7). The derivative of the vector $\phi(x)$ can be expressed by

$\phi^{\prime}(x)=\frac{d \phi(x)}{d x} \simeq \mathbf{D} \phi(x)$,

where $\mathbf{D}$ is $(N+1) \times(N+1)$ operational matrix of the derivative. Then, the nonzero elements $d_{k \ell}$ for $0 \leq k, \ell \leq N$ are given as follows:

$$
\begin{aligned}
d_{k+1, k} & =\frac{(\rho+k+1)(\rho+\sigma+2 k+1)}{L(\rho+\sigma+k+1)}, \quad d_{k k}=-\frac{k}{L}, \\
d_{k \ell} & =\frac{(-1)^{k+\ell+1}(2 \ell+\rho+\sigma+1)}{L} \prod_{r=1}^{k-\ell} \frac{(\rho+k-r+1)}{(\rho+\sigma+k-r+1)}, \quad \ell<k-1 .
\end{aligned}
$$

Corollary 4 (ChebyshevV Case) If $\rho=-\frac{1}{2}, \sigma=\frac{1}{2}$, then the nonzero elements $d_{k \ell}$ for $0 \leq k, \ell \leq N$ are given as follows:

$$
\begin{aligned}
d_{k+1, k} & =\frac{(2 k+1)^{2}}{2 L(k+1)}, \quad d_{k k}=-\frac{k}{L}, \\
d_{k \ell} & =\frac{(2 \ell+1)(-1)^{k+\ell+1} \Gamma(-k)\left(\frac{1}{2}-k\right)_{k-\ell}}{L \Gamma(-\ell)}, \quad \ell<k-1 .
\end{aligned}
$$

It easily noted that $\mathbf{D}$ is a lower-Heisenberg matrix.

Proof See, Bhrawy et al. [19].

Studying the class of exponential Jacobi functions yields many special orthogonal functions as a direct special cases, and these cases are reported in the following corollaries:

Corollary 1 (Legendre Case) If $\rho=\sigma=0$, then the nonzero elements, of the operational matrix of the exponential Legendre functions, $d_{k \ell}$ for $0 \leq k, \ell \leq N$ are given as follows:

$$
\begin{aligned}
d_{k+1, k} & =\frac{2 k+1}{L}, \quad d_{k k}=-\frac{k}{L}, \\
d_{k \ell} & =(-1)^{k+\ell+1} \frac{(2 \ell+1)}{L}, \quad \ell<k-1 .
\end{aligned}
$$

Corollary 2 (ChebyshevT Case) If $\rho=\sigma=-\frac{1}{2}$, then the nonzero elements, of the operational matrix of the exponential Chebyshev functions of the first kind, $d_{k \ell}$ for $0 \leq k, \ell \leq N$ are given as follows:

$$
\begin{aligned}
d_{k+1, k} & =\frac{2 k+1}{L}, \quad d_{k k}=-\frac{k}{L}, \\
d_{k \ell} & =\frac{2(-1)^{k+\ell+1} \ell\left(k-\frac{1}{2}\right)_{k-\ell}}{L(1-k)_{k-\ell}}, \quad \ell<k-1 .
\end{aligned}
$$

Corollary 3 (ChebyshevU Case) If $\rho=\sigma=\frac{1}{2}$, then the nonzero elements, of the operational matrix of the exponential Chebyshev functions of the second kind, $d_{k \ell}$ for $0 \leq k, \ell \leq N$ are given as follows:

$$
\begin{aligned}
d_{k+1, k} & =\frac{(2 k+3)(k+1)}{L(k+2)}, \quad d_{k k}=-\frac{k}{L}, \\
d_{k \ell} & =\frac{2(-1)^{k+\ell+1}(\ell+1)\left(k-\frac{1}{2}\right)_{k-\ell}}{L(-k-1)_{k-\ell}}, \quad \ell<k-1 .
\end{aligned}
$$

Corollary 5 (ChebyshevW Case) If $\rho=\frac{1}{2}, \sigma=-\frac{1}{2}$, then the nonzero elements $d_{k \ell}$ for $0 \leq k, \ell \leq N$ are given as follows:

$$
\begin{aligned}
d_{k+1, k} & =\frac{(2 k+3)(2 k+1)}{2 L(k+1)}, \quad d_{k k}=-\frac{k}{L}, \\
d_{k, \ell} & =\frac{2(-1)^{k+\ell} \Gamma(-k) \Gamma\left(\frac{1}{2}-\ell\right)}{L \Gamma\left(-k-\frac{1}{2}\right) \Gamma(-\ell)}, \quad \ell<k-1,
\end{aligned}
$$

Remark 1 The operational matrix for $r$-th derivative can be derived as

$\frac{d^{r} \phi(x)}{d x^{r}}=\left(\mathbf{D}^{(1)}\right)^{r} \phi(x)$,

where $r \in N$ and the superscript in $\mathbf{D}^{(1)}$ denote matrix powers. Thus,

$\mathbf{D}^{(r)}=\left(\mathbf{D}^{(1)}\right)^{r}, \quad r=1,2, \ldots$

\section{Implementation of the method}

The target of this part is to derive a scheme for the exponential Jacobi spectral collocation method based on the operational matrix of derivative of exponential Jacobi function to numerically solve the HPDEs on the half line. Let us consider the HPDEs of the form [25]

$\frac{\partial v(x, t)}{\partial t}=\xi_{1} \frac{\partial v(x, t)}{\partial x}+\xi_{2} v(x, t)+\mathcal{S}(x, t),(x, t) \in[0, \infty) \times[0, \infty)$,

subject to the initial conditions

$$
\begin{aligned}
& v(x, 0)=k_{0}(x), \quad x \in[0, \infty), \\
& v(0, t)=k_{1}(t), \quad t \in[0, \infty) .
\end{aligned}
$$


We approximate $v(x, t), \frac{\partial v(x, t)}{\partial t}$ and $\frac{\partial v(x, t)}{\partial x}$ by the double exponential Jacobi functions as

$$
\begin{aligned}
v(x, t) \approx v_{N, M}(x, t) & =\sum_{i=0}^{M} \sum_{j=0}^{N} c_{i j} \Upsilon_{i}^{\left(\rho_{1}, \sigma_{1}\right)}(x) \Upsilon_{j}^{\left(\rho_{2}, \sigma_{2}\right)}(t) \\
& =\phi_{N}(t) \mathbf{C}^{T} \phi_{M}(x), \\
\frac{\partial v_{N, M}(x, t)}{\partial t} & =\sum_{i=0}^{M} \sum_{j=0}^{N} c_{i j} \Upsilon_{i}^{\left(\rho_{1}, \sigma_{1}\right)}(x) \frac{\partial \Upsilon_{j}^{\left(\rho_{2}, \sigma_{2}\right)}(t)}{\partial t} \\
& =\phi_{N}^{\prime}(t) \mathbf{C}^{T} \phi_{M}(x), \\
\frac{\partial v_{N, M}(x, t)}{\partial x} & =\sum_{i=0}^{M} \sum_{j=0}^{N} c_{i j} \frac{\partial \Upsilon_{i}^{\left(\rho_{1}, \sigma_{1}\right)}(x)}{\partial x} \Upsilon_{j}^{\left(\rho_{2}, \sigma_{2}\right)}(t) \\
& =\phi_{N}(t) \mathbf{C}^{T} \phi_{M}^{\prime}(x),
\end{aligned}
$$

where $\mathbf{C}^{T}$ is $(N+1) \times(M+1)$ unknown matrix. Now, using Eqs. (14), (15) and (16), then it is easy to write

$\phi_{N}^{\prime}(t) \mathbf{C}^{T} \phi_{M}(x)=\xi_{1} \phi_{N}(t) \mathbf{C}^{T} \phi_{M}^{\prime}(x)+\xi_{2} \phi_{N}(t) \mathbf{C}^{T} \phi_{M}(x)+\mathcal{S}(x, t)$,

$\phi_{N}(0) \mathbf{C}^{T} \phi_{M}(x)=k_{0}(x)$,

$\phi_{N}(t) \mathbf{C}^{T} \phi_{M}(0)=k_{1}(t)$,

Now, we tame the collocation procedure for solving Eqs. (17)-(19). Suppose $x_{i}^{\left(\rho_{1}, \sigma_{1}\right)}(0 \leqslant i \leqslant M)$ are the exponential Jacobi collocation points of $\Upsilon_{i}^{\left(\rho_{1}, \sigma_{1}\right)}(x)$ and $t_{j}^{\left(\rho_{2}, \sigma_{2}\right)}(0 \leqslant j \leqslant N-1)$ are the exponential Jacobi collocation points of $\Upsilon_{j}^{\left(\rho_{2}, \sigma_{2}\right)}(t)$. We substitute these collocation points in (17)-(19); therefore, the collocation scheme can be written as:

$$
\begin{aligned}
& \phi_{N}^{\prime}\left(t_{j}^{\left(\rho_{2}, \sigma_{2}\right)}\right) \mathbf{C}^{T} \phi_{M}\left(x_{i}^{\left(\rho_{1}, \sigma_{1}\right)}\right)=\xi_{1} \phi_{N}\left(t_{j}^{\left(\rho_{2}, \sigma_{2}\right)}\right) \mathbf{C}^{T} \phi_{M}^{\prime}\left(x_{i}^{\left(\rho_{1}, \sigma_{1}\right)}\right) \\
& \quad+\xi_{2} \phi_{N}\left(t_{j}^{\left(\rho_{2}, \sigma_{2}\right)}\right) \mathbf{C}^{T} \phi_{M}\left(x_{i}^{\left(\rho_{1}, \sigma_{1}\right)}\right)+\mathcal{S}\left(x_{i}^{\left(\rho_{1}, \sigma_{1}\right)}, t_{j}^{\left(\rho_{2}, \sigma_{2}\right)}\right) \\
& 1 \leq i \leq M, 0 \leq j \leq N-1 .
\end{aligned}
$$

$\phi_{N}(0) \mathbf{C}^{T} \phi_{M}\left(x_{i}^{\left(\rho_{1}, \sigma_{1}\right)}\right)=k_{0}\left(x_{i}^{\left(\rho_{1}, \sigma_{1}\right)}\right), 0 \leq i \leq M$,

$\phi_{N}\left(t_{j}^{\left(\rho_{2}, \sigma_{2}\right)}\right) \mathbf{C}^{T} \phi_{M}(0)=k_{1}\left(t_{j}^{\left(\rho_{2}, \sigma_{2}\right)}\right), \quad 0 \leq j \leq N-1$.

This yields a algebraic system of $(N+1) \times(M+1)$ equations in the required double exponential Jacobi coefficients $c_{i j}, i=0,1, \ldots, M ; j=0,1, \ldots, N$, which can be solved by using any standard iteration technique, like Newton's iteration solver. Consequently, the approximate solution $v_{N, M}(x, t)$ can be evaluated.

\section{Error analysis}

Here, we discuss the convergence rate of the suggested double basis expansion, for this target, the following lemmas are needed:

Lemma 1 The following definite integral is valid:

$$
\begin{aligned}
& \int_{0}^{\infty} \Upsilon_{i}^{(\rho, \sigma)}(x) w^{(\rho+\mu+1, \sigma)} \\
& d x=\frac{L \Gamma(i+\sigma+1) \Gamma(\rho+\mu+1)(-\mu)_{i}}{i ! \Gamma(i+\rho+\sigma+\mu+2)} \\
& \rho+\mu>-1, \quad \sigma>-1,
\end{aligned}
$$

where $(a)_{i}$ denote the Pochhammer notation, i.e., $(a)_{i}=\Gamma(a+i) / \Gamma(a)$.

Lemma 2 For all $\rho>-1$, there exist two generic constants $0<\kappa_{1}<\kappa_{2}$ such that:

$\kappa_{1} n^{\rho-1} n ! \leq \Gamma(n+\rho) \leq \kappa_{2} n^{\rho-1} n ! ; \quad \forall n \in \mathbb{N}$.

Lemma 3 If $\rho, \sigma>-1$ then $\left|Y_{i}^{(\rho, \sigma)}(x)\right| \leq J / i^{q}$ where $q=\max \left(\rho, \sigma,-\frac{1}{2}\right)$, where $J$ is a generic positive constant.

In this theorem, we ascertain the vanishing rate of the unknown expansion coefficients of the approximate solution, under certain constrains on the exact smooth solution of the solved problem.

Theorem 2 If $v(x, t)$ is separable, i.e., $v(x, t)=v_{1}(x) v_{2}(t)$ and $v_{1}, v_{2}$ are of exponential order, in the sense that, there exist $A_{1}, A_{2}, \mu_{1}$ and $\mu_{2}$ positive constants, such that $\left|v_{1}(x)\right| \leq A_{1} e^{-\mu_{1} x}$ and $\left|v_{2}(t)\right| \leq A_{2} e^{-\mu_{2} t}$, then the expansion coefficients in (14) satisfy the following estimate:

$\left|c_{i j}\right| \leq \frac{C}{i^{\rho_{1}+2 \mu_{1}+1} j^{\rho_{2}+2 \mu_{2}+1}}$.

Proof By the hypothesis of theorem, we have,

$v(x, t)=v_{1}(x) v_{2}(t)=\sum_{m=0}^{\infty} \sum_{k=0}^{\infty} c_{k m} \Upsilon_{k}^{\left(\rho_{1}, \sigma_{1}\right)}(x) \Upsilon_{m}^{\left(\rho_{2}, \sigma_{2}\right)}(t)$,

applying the inner product, and by the orthogonality relation (3), we get,

$\left(v_{1}(x) v_{2}(t), \Upsilon_{i}^{\left(\rho_{1}, \sigma_{1}\right)}(x) \Upsilon_{j}^{\left(\rho_{2}, \sigma_{2}\right)}(t)\right)_{w^{\left(\rho_{1}, \sigma_{1}\right)} w_{w^{\left(\rho_{2}, \sigma_{2}\right)}}}=c_{i j} h_{i}^{\left(\rho_{1}, \sigma_{1}\right)} h_{j}^{\left(\rho_{2}, \sigma_{2}\right)}$,

i.e., 


$$
\begin{aligned}
c_{i j}= & \frac{1}{h_{i}^{\left(\rho_{1}, \sigma_{1}\right)} h_{j}^{\left(\rho_{2}, \sigma_{2}\right)}} \int_{0}^{\infty} \int_{0}^{\infty} v(x, t) \Upsilon_{i}^{\left(\rho_{1}, \sigma_{1}\right)}(x) \Upsilon_{j}^{\left(\rho_{2}, \sigma_{2}\right)}(t) \\
& w^{\left(\rho_{1}, \sigma_{1}\right)} w^{\left(\rho_{2}, \sigma_{2}\right)} d x d t \\
= & \frac{1}{h_{i}^{\left(\rho_{1}, \sigma_{1}\right)} h_{j}^{\left(\rho_{2}, \sigma_{2}\right)}}\left(\int_{0}^{\infty} v_{1}(x) \Upsilon_{i}^{\left(\rho_{1}, \sigma_{1}\right)}(x) w^{\left(\rho_{1}, \sigma_{1}\right)} d x\right) \\
& \left(\int_{0}^{\infty} v_{2}(t) \Upsilon_{j}^{\left(\rho_{2}, \sigma_{2}\right)}(t) w^{\left(\rho_{2}, \sigma_{2}\right)} d t\right) \\
= & I_{1}^{\left(\rho_{1}, \sigma_{1}\right)}(i) I_{2}^{\left(\rho_{2}, \sigma_{2}\right)}(j)
\end{aligned}
$$

where,

$I_{r}^{\left(\rho_{r}, \sigma_{r}\right)}(k)=\frac{1}{h_{k}^{\left(\rho_{r}, \sigma_{r}\right)}} \int_{0}^{\infty} v_{r}(z) \Upsilon_{k}^{\left(\rho_{r}, \sigma_{r}\right)}(z) w^{\left(\rho_{r}, \sigma_{r}\right)} d z, \quad r=1,2$.

Now by application of integration by parts on $I_{1}^{\left(\rho_{1}, \sigma_{1}\right)}(i)$ and $I_{2}^{\left(\rho_{2}, \sigma_{2}\right)}(j)$, since $v_{1}$ and $v_{2}$ are of exponential order, by the integral formula in Lemma 1 , repeated use of the estimate in Lemma 2 on $I_{1}^{\left(\rho_{1}, \sigma_{1}\right)}(i)$ and $I_{2}^{\left(\rho_{2}, \sigma_{2}\right)}(j)$, the theorem is proved.

In this theorem, based on the result of the previous theorem, we ascertain the convergence of the approximate solution as the number of retained modes increases.

Theorem 3 If $\min \left(\rho_{1}+2 \mu_{1}, \rho_{2}+2 \mu_{2}\right)>\frac{1}{2} \quad$ and $-1<\max \left(\rho_{1}, \rho_{2}, \sigma_{1}, \sigma_{2}\right)<-\frac{1}{2}$, then series in (14) converges absolutely.

Proof We show that the series $\left|\sum_{0}^{\infty} \sum_{0}^{\infty} c_{i j} \Upsilon_{i}^{\left(\rho_{1}, \sigma_{1}\right)}(x) \Upsilon_{j}^{\left(\rho_{2}, \sigma_{2}\right)}(t)\right|$ converges absolutely.

By the estimate in Theorem 2, using Lemma 3, then

$\left|c_{i j} \Upsilon_{i}^{\left(\rho_{1}, \sigma_{1}\right)}(x) \Upsilon_{j}^{\left(\rho_{2}, \sigma_{2}\right)}(t)\right| \leq \frac{A}{i^{\rho_{1}+2 \mu_{1}+\frac{1}{2}} j^{\rho_{2}+2 \mu_{2}+\frac{1}{2}}}$,

which completes the proof of the theorem.

In this theorem, we control the estimate of two consecutive approximate solutions, to ascertain the stability when the number of retained modes increases.

Theorem 4 If $\min \left(\rho_{1}+2 \mu_{1}, \rho_{2}+2 \mu_{2}\right)>\frac{1}{4} \quad$ and $-1<\max \left(\rho_{1}, \rho_{2}, \sigma_{1}, \sigma_{2}\right)<-\frac{1}{2}$, then

$\lim _{N, M \rightarrow \infty}\left\|u_{N+1, M+1}-u_{N, M}\right\|_{2}=0$.

Proof By the triangle inequality, we have,

$$
\begin{aligned}
\left\|u_{N+1, M+1}-u_{N, M}\right\|_{2}=\left\|u_{N+1, M+1}-u_{N, M+1}+u_{N, M+1}-u_{N, M}\right\|_{2} \\
\leq \\
=\left\|u_{N+1, M+1}-u_{N, M+1}\right\|_{2}+\left\|u_{N, M+1}-u_{N, M}\right\|_{2} \\
\quad+\left\|\sum_{j=0}^{M+1} c_{N+1, j} \Upsilon_{N+1}^{\left(\rho_{1}, \sigma_{1}\right)}(x) \Upsilon_{j}^{\left(\rho_{2}, \sigma_{2}\right)}(t)\right\|_{2} c_{i, M+1} \Upsilon_{i}^{\left(\rho_{1}, \sigma_{1}\right)}(x) \Upsilon_{M+1}^{\left(\rho_{2}, \sigma_{2}\right)}(t) \|_{2} .
\end{aligned}
$$

Now, application of Lemma 2, Lemma 3 to the two norms of the R.H.S of the later inequality, respectively, and by the result of Theorem 3, we get

$\left\|u_{N+1, M+1}-u_{N, M}\right\|_{2}<\frac{B}{M^{2 \rho_{1}+4 \mu_{1}-\frac{1}{2}} N^{2 \rho_{2}+4 \mu_{2}-\frac{1}{2}}}$,

which completes the proof of the theorem.

\section{Numerical results}

In this section, we test our algorithm by exibiting two numerical experiments to check the applicability and accuracy of the proposed scheme. Comparison of the numerical results obtained by the suggested technique with those obtained by generalized Laguerre-Gauss-Radau collocation approach [25] confirms that the presented scheme is very effective and convenient. Thereby, we assert that the proposed scheme is more appropriate for solving these kinds of problems.

The absolute errors in the given tables are

$E(x, t)=\left|v(x, t)-v_{N, M}(x, t)\right|$

where $v(x, t)$ and $v_{N, M}(x, t)$ are the exact solution and the numerical solution, respectively, at the point $(x, t)$, respectively. Moreover, the maximum absolute errors are given by

$L^{\infty}=\operatorname{Max}\{E(x, t): \forall(x, t) \in[0, \infty) \times[0, \infty)\}$.

Example 1 [25] Consider the hyperbolic equation of firstorder of the form

$\frac{\partial v(x, t)}{\partial t}=\frac{\partial v(x, t)}{\partial x}+v(x, t)+\mathcal{S}(x, t), \quad, x \in[0, \infty), \quad t \in[0, \infty)$,

subject to initial conditions,

$v(x, 0)=e^{-x}, \quad x \in[0, \infty), \quad v(0, t)=e^{-\sqrt{2} t}, \quad t \in[0, \infty)$, 
Table 1 Comparison of the absolute errors for Example 1 at $t=0.1$ and $N=M=16$

\begin{tabular}{lllll}
\hline$x$ & Bhrawy et al. [25] & Our method \\
\cline { 3 - 5 } & & $\rho_{1}=\sigma_{1}=-\frac{1}{2}$, & $\rho_{1}=\sigma_{1}=0$ & $\rho_{1}=\sigma_{1}=\frac{1}{2}$ \\
& & $\rho_{2}=\sigma_{2}=-\frac{1}{2}$ & $\rho_{2}=0$ & \\
& & & $\rho_{2}=\sigma_{2}=\frac{1}{2}$ \\
\hline 0.1 & $2.84 \times 10^{-7}$ & $2.29 \times 10^{-8}$ & $1.04 \times 10^{-8}$ & $2.97 \times 10^{-8}$ \\
0.2 & $8.79 \times 10^{-6}$ & $1.84 \times 10^{-8}$ & $9 \times 10 \times 10^{-9}$ & $2.64 \times 10^{-9}$ \\
0.3 & $1.20 \times 10^{-5}$ & $1.84 \times 10^{-8}$ & $8.57 \times 10^{-9}$ & $2.45 \times 10^{-9}$ \\
0.4 & $1.12 \times 10^{-5}$ & $1.48 \times 10^{-8}$ & $7.47 \times 10^{-9}$ & $2.15 \times 10^{-9}$ \\
0.5 & $7.95 \times 10^{-6}$ & $1.51 \times 10^{-8}$ & $6.98 \times 10^{-9}$ & $2.02 \times 10^{-9}$ \\
0.6 & $3.29 \times 10^{-6}$ & $1.33 \times 10^{-8}$ & $6.29 \times 10^{-9}$ & $1.79 \times 10^{-9}$ \\
0.7 & $1.78 \times 10^{-6}$ & $1.08 \times 10^{-8}$ & $5.51 \times 10^{-9}$ & $1.58 \times 10^{-9}$ \\
0.8 & $6.53 \times 10^{-6}$ & $1.05 \times 10^{-8}$ & $5.08 \times 10^{-9}$ & $1.48 \times 10^{-9}$ \\
0.9 & $1.04 \times 10^{-5}$ & $1.06 \times 10^{-8}$ & $4.75 \times 10^{-9}$ & $1.37 \times 10^{-9}$ \\
1 & $1.32 \times 10^{-5}$ & $9.25 \times 10^{-9}$ & $4.24 \times 10^{-9}$ & $1.20 \times 10^{-9}$ \\
\hline
\end{tabular}

Table 2 Comparison of the absolute errors for Example 1 at $t=0.5$ and $N=M=16$

\begin{tabular}{lllll}
\hline$x$ & Bhrawy et al. [25] & Our method & & \\
\cline { 3 - 5 } & & $\rho_{1}=\sigma_{1}=-\frac{1}{2}$, & $\rho_{1}=\sigma_{1}=0$ & $\rho_{1}=\sigma_{1}=\frac{1}{2}$, \\
& & $\rho_{2}=\sigma_{2}=-\frac{1}{2}$ & $\rho_{2}=\sigma_{2}=0$ & \\
& & $\rho_{2}=\sigma_{2}=\frac{1}{2}$ \\
\hline 0.1 & $8.95 \times 10^{-6}$ & $7.65 \times 10^{-8}$ & $3.54 \times 10^{-8}$ & $1.50 \times 10^{-8}$ \\
0.2 & $4 \times 10 \times 10^{-6}$ & $6.22 \times 10^{-8}$ & $3.09 \times 10^{-8}$ & $1.34 \times 10^{-8}$ \\
0.3 & $1.39 \times 10^{-5}$ & $6.16 \times 10^{-8}$ & $2.90 \times 10^{-8}$ & $1.23 \times 10^{-8}$ \\
0.4 & $2.07 \times 10^{-5}$ & $5.04 \times 10^{-8}$ & $2.54 \times 10^{-8}$ & $1 \times 10 \times 10^{-8}$ \\
0.5 & $2.47 \times 10^{-5}$ & $5.05 \times 10^{-8}$ & $2.36 \times 10^{-8}$ & $1.00 \times 10^{-8}$ \\
0.6 & $2.62 \times 10^{-6}$ & $4.48 \times 10^{-8}$ & $2.13 \times 10^{-8}$ & $9.13 \times 10^{-9}$ \\
0.7 & $2.55 \times 10^{-5}$ & $3.68 \times 10^{-8}$ & $1.87 \times 10^{-8}$ & $8.19 \times 10^{-9}$ \\
0.8 & $2.31 \times 10^{-5}$ & $3.53 \times 10^{-8}$ & $1.72 \times 10^{-8}$ & $7.45 \times 10^{-9}$ \\
0.9 & $1.93 \times 10^{-5}$ & $3.51 \times 10^{-8}$ & $1.61 \times 10^{-8}$ & $6.80 \times 10^{-9}$ \\
1 & $1.45 \times 10^{-5}$ & $3.08 \times 10^{-8}$ & $1.43 \times 10^{-8}$ & $6.11 \times 10^{-9}$ \\
& & & &
\end{tabular}

where

$\mathcal{S}(x, t)=-\sqrt{2} e^{-\sqrt{2} t-x}$.

The exact solution is given by

$v(x, t)=e^{-(\sqrt{2} t+x)}$.

In Tables 1, 2 and 3, we give the absolute errors with $\rho_{1}=\sigma_{1}=\rho_{2}=\sigma_{2}=-\frac{1}{2}$ (first kind exponential Chebyshev functions), $\rho_{1}=\sigma_{1}=\stackrel{\rho}{\rho}_{2}=\sigma_{2}=0$ (exponential Legendre functions) and $\rho_{1}=\sigma_{1}=\rho_{2}=\sigma_{2}=\frac{1}{2}$ (second kind exponential Chebyshev functions), respectively, at $N=M=16$.
Table 3 Comparison of the absolute errors for Example 1 at $t=1$ and $N=M=16$

\begin{tabular}{lllll}
\hline$x$ & Bhrawy et al. [25] & Our method & \\
\cline { 3 - 5 } & & $\rho_{1}=\sigma_{1}=-\frac{1}{2}$, & $\rho_{1}=\sigma_{1}=0$ & $\rho_{1}=\sigma_{1}=\frac{1}{2}$ \\
& & $\rho_{2}=\sigma_{2}=-\frac{1}{2}$ & $\rho_{2}=0$ & \\
& & & $\rho_{2}=\sigma_{2}=\frac{1}{2}$ \\
\hline 0.1 & $4.87 \times 10^{-5}$ & $1.71 \times 10^{-8}$ & $2.47 \times 10^{-8}$ & $2.19 \times 10^{-8}$ \\
0.2 & $4.89 \times 10^{-5}$ & $2.37 \times 10^{-8}$ & $2.34 \times 10^{-8}$ & $1.99 \times 10^{-8}$ \\
0.3 & $4.17 \times 10^{-5}$ & $1.51 \times 10^{-8}$ & $2.02 \times 10^{-8}$ & $1.79 \times 10^{-8}$ \\
0.4 & $3.03 \times 10^{-5}$ & $1.99 \times 10^{-8}$ & $1.91 \times 10^{-8}$ & $1.63 \times 10^{-8}$ \\
0.5 & $1.75 \times 10^{-5}$ & $1.24 \times 10^{-8}$ & $1.66 \times 10^{-8}$ & $1.47 \times 10^{-8}$ \\
0.6 & $4.74 \times 10^{-6}$ & $1.21 \times 10^{-8}$ & $1.51 \times 10^{-8}$ & $1.33 \times 10^{-8}$ \\
0.7 & $6.68 \times 10^{-6}$ & $1.53 \times 10^{-8}$ & $1.42 \times 10^{-8}$ & $1.21 \times 10^{-8}$ \\
0.8 & $1.61 \times 10^{-5}$ & $1.16 \times 10^{-8}$ & $1.26 \times 10^{-8}$ & $1.09 \times 10^{-8}$ \\
0.9 & $2.32 \times 10^{-5}$ & $6.90 \times 10^{-9}$ & $1.09 \times 10^{-8}$ & $9.79 \times 10^{-9}$ \\
1 & $2.79 \times 10^{-5}$ & $7.26 \times 10^{-9}$ & $1.00 \times 10^{-8}$ & $8.87 \times 10^{-9}$ \\
\hline & & & &
\end{tabular}

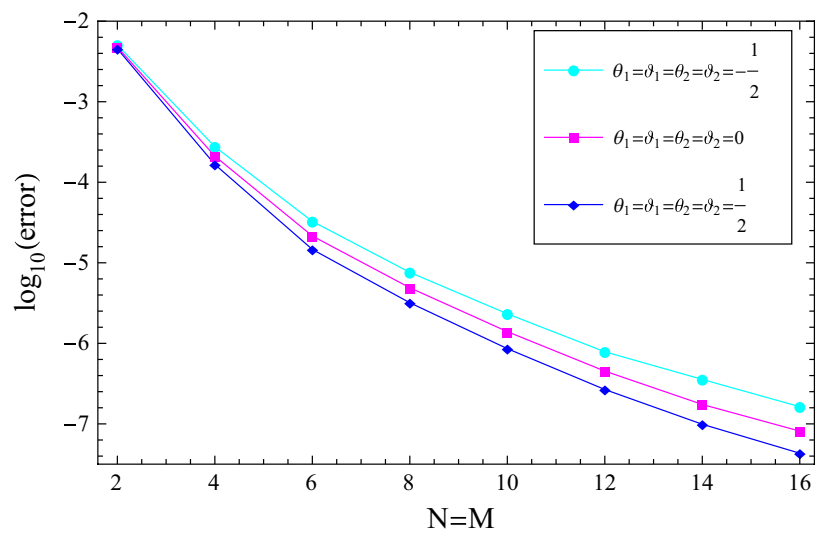

Fig. $1 L^{\infty}$ error for Example 1 versus $N=M$ and $\rho_{1}=\sigma_{1}=\rho_{2}=\sigma_{2}$

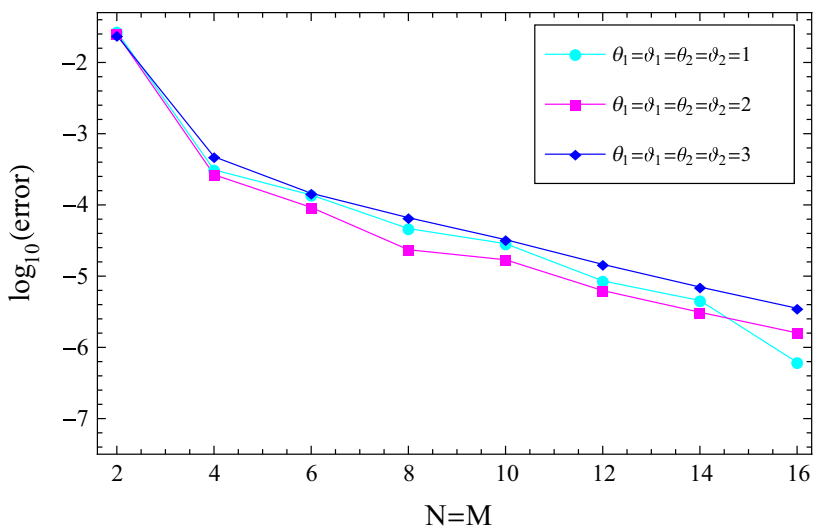

Fig. $2 L^{\infty}$ error for Example 2 versus $N=M$ and $\rho_{1}=\sigma_{1}=\rho_{2}=\sigma_{2}$ 
Table 4 The absolute errors for Example 2 at $N=M=16$

\begin{tabular}{llll}
\hline$(x, t)$ & $\rho_{1}=\sigma_{1}=-\frac{1}{2}$, & $\begin{array}{l}\rho_{1}=\sigma_{1}=0, \\
\rho_{2}=\sigma_{2}=0\end{array}$ & $\begin{array}{l}\rho_{1}=\sigma_{1}=\frac{1}{2}, \\
\\
\rho_{2}=\sigma_{2}=-\frac{1}{2}\end{array}$ \\
\hline$(0.1,0.1)$ & $9.61 \times 10^{-7}$ & $4.22 \times 10^{-7}$ & $1.95 \times 10^{-7}$ \\
$(0.2,0.2)$ & $6.37 \times 10^{-7}$ & $5.92 \times 10^{-7}$ & $7.08 \times 10^{-7}$ \\
$(0.3,0.3)$ & $9.63 \times 10^{-7}$ & $6.47 \times 10^{-7}$ & $1.67 \times 10^{-7}$ \\
$(0.4,0.4)$ & $1.44 \times 10^{-6}$ & $8.90 \times 10^{-7}$ & $7.52 \times 10^{-7}$ \\
$(0.5,0.5)$ & $2.04 \times 10^{-6}$ & $9.36 \times 10^{-7}$ & $2.47 \times 10^{-7}$ \\
$(0.6,0.6)$ & $1.28 \times 10^{-8}$ & $4.81 \times 10^{-8}$ & $1.35 \times 10^{-7}$ \\
$(0.7,0.7)$ & $2.23 \times 10^{-6}$ & $1.23 \times 10^{-6}$ & $8.48 \times 10^{-7}$ \\
$(0.8,0.8)$ & $7.97 \times 10^{-7}$ & $4.56 \times 10^{-7}$ & $1.16 \times 10^{-7}$ \\
$(0.9,0.9)$ & $3.33 \times 10^{-6}$ & $1.55 \times 10^{-6}$ & $6.54 \times 10^{-7}$ \\
\hline
\end{tabular}

Table 5 Comparison of the maximum absolute errors for Example 2 versus $\rho_{1}=\sigma_{1}, \rho_{2}=\sigma_{2}$ at $N=M=16$

\begin{tabular}{llll}
\hline$\rho_{1}=\sigma_{1}$ & $\rho_{2}=\sigma_{2}$ & Bhrawy et al. [25] & Our method \\
\hline 1 & 1 & $6.00 \times 10^{-5}$ & $6.16 \times 10^{-7}$ \\
2 & 2 & $2.56 \times 10^{-4}$ & $1.59 \times 10^{-6}$ \\
3 & 3 & $3.51 \times 10^{-4}$ & $3.53 \times 10^{-6}$ \\
\hline
\end{tabular}

Moreover, the results obtained by our method are compared with these obtained by generalized Laguerre-Gauss-Radau collocation method [25]. Figure 1 shows $L^{\infty}$ error versus $N=M$ and $\rho_{1}=\sigma_{1}=\rho_{2}=\sigma_{2}$.

Example 2 [25] Consider the following hyperbolic equation of first-order

$$
\begin{gathered}
\frac{\partial v(x, t)}{\partial t}=\frac{\partial v(x, t)}{\partial x}+v(x, t)+e^{-t-x}(\cos (t)-\sin (t)), x \in[0, \infty), \\
t \in[0, \infty),
\end{gathered}
$$

subject to initial conditions,

$v(x, 0)=0, \quad x \in[0, \infty), \quad v(0, t)=e^{-t} \sin (t), \quad t \in[0, \infty)$.

The exact solution is given by

$v(x, t)=e^{-(t+x)} \sin (t)$.

Table 4 lists the results obtained by the our method in terms of absolute errors at $N=M=16$ for different values of $\rho_{1}, \sigma_{1}, \rho_{2}, \sigma_{2}, x$ and $t$. Figure 2 shows the $L^{\infty}$ error versus $\rho_{1}=\sigma_{1}=\rho_{2}=\sigma_{2}$ and $N=M$. Moreover, the results in Table 5 are more accurate if compared with these obtained by generalized Laguerre-Gauss-Radau collocation method [25].

\section{Conclusion}

We developed an accurate numerical technique and applied it to solve hyperbolic partial differential equations. The proposed operational matrix in combination with the exponential Jacobi spectral-collocation approach was elaborated for reducing the solution of hyperbolic first-order partial differential equations on the semi-infinite domain to an algebraic system of equations, which can be solved more easily. The operational matrices of derivatives of exponential Legendre, ChebyshevT, U, V, W functions can be obtained as direct special cases of the operational matrix of exponential Jacobi functions. The numerical results evince the high efficiency and accuracy of our approach.

Acknowledgements The authors are very grateful to the anonymous referees for careful reviewing and crucial comments, which enabled us to improve the manuscript.

Open Access This article is distributed under the terms of the Creative Commons Attribution 4.0 International License (http://creativeco mmons.org/licenses/by/4.0/), which permits unrestricted use, distribution, and reproduction in any medium, provided you give appropriate credit to the original author(s) and the source, provide a link to the Creative Commons license, and indicate if changes were made.

\section{References}

1. Jordan, P.M., Puri, A.: Digital signal propagation in dispersive media. J. Appl. Phys. 85, 1273-1282 (1999)

2. Weston, V.H., He, S.: Wave splitting of the telegraph equation in R3 and its application to inverse scattering. Inverse Probl. 9, 789-812 (1993)

3. Yu, S.T.J., Yang, L., Lowe, R.L., Bechtel, S.E.: Numerical simulation of linear and nonlinear waves in hypoelastic solids by the CESE method. Wave Motion 47, 168-182 (2010)

4. Bonazzola, S., Gourgoulhon, E., Marck, J.-A.: Spectral methods in general relativistic astrophysics. J. Comput. Appl. Math. 109, 433-473 (1999)

5. Zhang, T., Tadé, M.O., Tian, Y.-C., Zang, H.: High-resolution method for numerically solving PDEs in process engineering. Comput. Chem. Eng. 32, 2403-2408 (2008)

6. Oberguggenberger, M.: Hyperbolic systems with discontinuous coefficients: generalized solutions and a transmission problem in acoustics. J. Math. Anal. Appl. 142, 452-467 (1989)

7. Banasiak, J., Mika, J.R.: Singularly perturbed telegraph equations with applications in the random walkt heory. J. Appl. Math. Stoch. Anal. 11, 9-28 (1998)

8. Lakestani, M., Sarray, B.N.: Numerical solution of telegraph equation using interpolating scaling function. Comput. Math. Appl. 60, 1964-1972 (2010) 
9. Mittal, R.C., Bhatia, R.: A numerical study of two dimensional hyperbolic telegraph equation by modifed B-spline differential quadrature method. Appl. Math. Comput. 244, 976-997 (2014)

10. Abd-Elhameed, W.M., Doha, E.H., Youssri, Y.H., Bassuony, M.A.: New Tchebyshev-Galerkin operational matrix method for solving linear and nonlinear hyperbolic telegraph type equations. Numer. Methods Partial Differ. Equ. 32(6), 1553-1571 (2016)

11. Doha, E.H., Abd-Elhameed, W.M., Youssri, Y.H.: Fully Legendre spectral Galerkin algorithm for solving linear onedimensional telegraph type equation. Int. J. Comput. Methods 16(8), 1850118 (2019)

12. Youssri, Y.H., Abd-Elhameed, W.M.: Numerical spectral Legendre-Galerkin algorithm for solving time fractional Telegraph equation. Rom. J. Pys. 63(3-4), 107 (2018)

13. Mu, L., Ye, X.: A simple finite element method for linear hyperbolic problems. J. Comput. Appl. Math. 330, 330-339 (2018)

14. Qin, X., Duan, X., Hu, G., Su, L., Wang, X.: An element-free Galerkin method for solving the two-dimensional hyperbolic problem. Appl. Math. Comput. 321, 106-120 (2018)

15. Hafez, R.M.: Numerical solution of linear and nonlinear hyperbolic telegraph type equations with variable coefficients using shifted Jacobi collocation method. Comput. Appl. Math. 37(4), 5253-5273 (2018)

16. Doha, E.H., Bhrawy, A.H., Hafez, R.M., Gorder, R.A.V.: A Jacobi rational pseudospectral method for Lane-Emden initial value problems arising in astrophysics on a semi-infinite interval. Comput. Appl. Math. 33, 607-619 (2014)

17. Hafez, R.M., Abdelkawy, M.A., Doha, E.H., Bhrawy, A.H.: A new collocation scheme for solving hyperbolic equations of second order in a semi-infinite domain. Rom. Rep. Phys. 68, 112-127 (2016)

18. Doha, E.H., Bhrawy, A.H., Hafez, R.M.: Numerical algorithm for solving multi-pantograph delay equations on the half-line using Jacobi rational functions with convergence analysis. Acta Math. Appl. Sin. Engl. Ser. 33, 297-310 (2017)

19. Bhrawy, A.H., Hafez, R.M., Alzaidy, J.F.: A new exponential Jacobi pseudospectral method for solving high-order ordinary differential equations. Adv. Differ. Equ. 2015, 152 (2015)

20. Bhrawy, A.H., Doha, E.H., Baleanu, D., Hafez, R.M.: A highly accurate Jacobi collocation algorithm for systems of high-order linear differential-difference equations with mixed initial conditions. Math. Methods Appl. Sci. 38, 3022-3032 (2015)

21. Hafez, R.M., Ezz-Eldien, S.S., Bhrawy, A.H., Ahmed, E.A., Baleanu, D.: A Jacobi Gauss-Lobatto and Gauss-Radau collocation algorithm for solving fractional Fokker-Planck equations. Nonlinear Dyn. 82, 1431-1440 (2015)

22. Bhrawy, A.H., Zaky, M.A.: An improved collocation method for multi-dimensional space-time variable-order fractional Schrödinger equations. Appl. Numer. Math. 111, 197-218 (2017)

23. Doha, E.H., Bhrawy, A.H., Hafez, R.M., Van Gorder, R.A.: Jacobi rational-Gauss collocation method for Lane-Emden equations of astrophysical significance. Nonlinear Anal. Model. Control 19, 537-550 (2014)

24. Bhrawy, A.H., Doha, E.H., Abdelkawy, M.A., Hafez, R.M.: An efficient collocation algorithm for multidimensional wave type equations with nonlocal conservation conditions. Appl. Math. Model. 39, 5616-5635 (2015)

25. Bhrawy, A.H., Hafez, R.M., Alzahrani, E.O., Baleanu, D., Alzahrani, A.A.: Generalized Laguerre-Gauss-Radau scheme for first order hyperbolic equations on semi-infinite domains. Rom. J. Phys. 60, 918-934 (2015)

Publisher's Note Springer Nature remains neutral with regard to jurisdictional claims in published maps and institutional affiliations. 